\title{
Factors Influencing Quality of Life in Patients with Gastroesophageal Reflux Disease in a Tertiary Care Hospital in Pakistan
}

\author{
Ghulamullah Lail1, Farina Muhammad Hanif1, Amanullah Lail2, Muhammad Manzoor ul Haque1, Abbas Ali Tasneem¹ \\ and Nasir Hassan Luck1 \\ ${ }^{1}$ Department of Hepatogastroenterology, Sindh Institute of Urology and Transplantation (SIUT), Karachi, Pakistan \\ 2Department of Pediatrics, Dow University Hospital, Karachi, Pakistan
}

\begin{abstract}
Objective: To assess the impact of gastroesophageal reflux disease (GERD) on quality of life (QOL) in local population. Study Design: Cross-sectional, descriptive study.

Place and Duration of Study: Hepatogastroenterology Department, Sindh Institute of Urology and Transplantation, Karachi, from June 2016 to December 2017.

Methodology: Patients with GERD visiting the outpatients' clinics were enrolled. Those who had history of dysphagia, malignant disease, anemia, weight loss, cardiovascular diseases, renal failure, cirrhosis, rheumatologic diseases and patients taking non-steroidal anti-inflammatory drugs (NSAIDS) or steroids during the last two weeks were exluded. A predesigned questionnaire was used to calculate GERD impact scale (GIS) score, with a value of $>18$ being considered as impaired QOL. Chi-square test was used to identify factors associated with impaired QOL.

Results: A total of 782 patients with mean age of $37.3 \pm 8.99$ years (range: $18-65$ years) were studied. Majority (505, $64.5 \%$ ) were younger than 40 years of age. Predominant study population were females, i.e. 413 (52.8\%). Among these, $127(16.2 \%)$ patients had symptoms lasting more than a year, 132 (16.9\%) were smokers, 82 (10.5\%) had diabetes mellitus (DM), and $63(8.1 \%)$ had hypertension. A total of $297(38 \%)$ patients had impaired QOL. On logistic regression analysis, the impaired QOL was associated with age greater than 40 years $(p=0.001)$, body mass index $(B M I)>25 \mathrm{Kg} / \mathrm{m}^{2}$ $(p=0.001)$, smoking $(p=0.001)$, hypertension $(p=0.001)$ and diabetes mellitus $(p=0.001)$.

Conclusion: A significant proportion of patients had impaired QOL due to GERD. Factors affecting QOL in such patients were higher age, increased BMI, history of smoking, hypertension and DM.
\end{abstract}

Key Words: Gastroesophageal reflux disease, Quality of life, Questionnaire, Heartburn.

How to cite this article: Lail G, Hanif FM, Lail A, Haque MM, Tasneem AA, Luck NH. Factors influencing quality of life in patients with gastroesophageal reflux disease in a tertiary care hospital in Pakistan. J Coll Physicians Surg Pak 2019; 29(9):882-5.

\section{INTRODUCTION}

Gastroesophageal reflux disease (GERD) is one of the most common diseases seen by the gastroenterologists as well as primary care physicians. ${ }^{1}$ It is a chronic condition in which reflux of stomach contents leads to esophageal and extra-esophageal symptoms. ${ }^{2}$ Heartburn and regurgitation are common symptoms seen in the general population, with $15-20 \%$ experiencing these symptoms weekly. ${ }^{2}$ The prevalence of GERD varies in different parts of the world; this is primarily based upon estimates rather than actual data. ${ }^{3} \mathrm{~A}$ study reviewing endoscopic reports from nearly 2.5 thousand consecutive patients, found complicated GERD in $12 \%$ of White, $3 \%$ of African-

Correspondence to: Dr. Ghulamullah Lail, Department of Hepatogastroenterology, Sindh Institute of Urology and Transplantation (SIUT), Karachi, Pakistan

E-mail: ga.lail@hotmail.com

Received: June 23, 2018; Revised: January 28, 2019;

Accepted: March 18, 2019
American and $2 \%$ of Asian patients. ${ }^{4}$ Its prevalence in Pakistan is reported to be around $24-35 \% .5,6$ Patients with troublesome GERD (daily or weekly symptoms) have an increase in time off work and decrease in physical functioning. ${ }^{7}$

Quality of life (QOL) is a broad multi-dimensional concept that usually includes subjective evaluations of both positive and negative aspects of life. ${ }^{8} \mathrm{WHO}$ defines QOL as an individual's perception of their position in life in the context of the culture and value systems in which they live and in relation to their goals, expectations, standards and concerns. Effect of GERD on QOL has been evaluated in different studies using questionnaires. The thirty-six-item short-form (SF-36) general health survey, is a self-reported, generic health-related QOL (HRQOL) survey widely used in various chronic diseases including GERD.9

Gastrooesophageal Reflux Disease Impact Scale (GIS) questionnaire is comprised of 9 items including 5 questions on GERD symptoms (chest pain, heartburn, 
acid regurgitation, epigastric pain and hoarseness) and 4 questions on QOL. This scale was shown to demonstrate internal consistency, reproducibility and concept validity in an earlier study. 10

GERD and its gastrointestinal and extra-intestinal symptoms can affect patient's QOL.8,11-14 Many studies from West and Asian countries validated effect of GERD on QOL.11,14,15 Evaluation of the disease-specific questionnaires in the field of reflux disease may show the impact of GERD on HR-QOL more accurately. ${ }^{16}$

There are relatively few studies on the impact of GERD on QOL of patients in Pakistan. Moreover, there is scant data on factors leading to poor QOL on patients with GERD. This study was designed to determine the impact of GERD on QOL and to identify factors leading to poor QOL in patients with GERD.

\section{METHODOLOGY}

This questionnaire-based cross-sectional study was conducted in the gastrointestinal (GI) outpatients' department of Sindh Institute of Urology and Transplantation, Karachi, from June 2016 to December 2017. A total of 782 patients of both genders, between 18 and 65 years of age, having one of the following symptoms for more than twice a week: presence of heartburn (retrosternal burning sensation) and/or regurgitation occurring in the last four weeks, were included in the study. These questionnaires were administered as per nonprobability, consecutive sampling from those patients who visited the OPD during this period. We assessed QOL using Urdu version of GIS. Median value of GIS score was 18; thus, a score $>18$ was labelled as impaired QOL.

Those excluded from the study were either those who failed to give consent, with history of dysphagia, malignant disease, anemia, weight loss, cardiovascular diseases, renal failure, cirrhosis, rheumatologic diseases and patients taking drugs like non-steroidal anti-inflammatory drugs (NSAIDS) or steroids during last two weeks. Ethical approval was sought from the Institutional Ethics Committee. Written informed consent was obtained from all patients fulfilling inclusion criteria. A structured proforma was used to collect data and a GIS questionnaire was filled by the researchers. Upper GI endoscopy was not performed in the study population.

The collected data was transferred to Statistical Package for the Social Sciences (SPSS) version 20.0 for the statistical analysis. QOL (impaired/unimpaired) was taken as outcome variable. Mean and standard deviation were calculated for continuous variables, while frequency and percentage were calculated for categorical variables. Stratification with respect to age, gender, duration of symptoms, BMI, DM, HTN and smoking status were made. Post-stratification, Chi-square test was applied. A $p$-value of $\leq 0.05$ was taken as statistically significant.
Multiple regression analyses were carried out on all factors that were significant on univariate analysis to assess the correlation.

\section{RESULTS}

A total of 782 patients with mean age of $37.35 \pm 8.99$ years were enrolled (Table I). Patients were predominantly females 413 (52.8\%). Regarding age, 505 patients (64.5\%) were younger than 40 years of age. Majority were Urdu-speaking, 360 (46\%); followed by Sindhis, 140 (17.9\%); Punjabis, 103 (13.2\%); Pathans, 73 (9.3\%); Balochis, 80 (10.2\%); and others, 26 (3.3\%). Regarding duration of symptoms, 127 (16.2\%) patients had symptoms lasting more than a year. Among all, 132 $(16.9 \%)$ patients were smokers, $82(10.5 \%)$ had diabetes mellitus (DM), and $63(8.1 \%)$ had hypertension.

On the basis of GIS questionnaire, impaired QOL was documented in 297 (38\%) patients. Stratification and correlation with respect to age, gender, duration of symptoms, BMI, DM, HTN and smoking status was performed to assess the associated factors impairing QOL in GERD patients (Table II). Impaired QOL was

Table I: Baseline characteristics of the study population.

\begin{tabular}{lc}
\hline Total $n u m b e r$ & $\mathrm{n}=782$ \\
\hline Males, $\mathrm{n}(\%)$ & $369(47.16 \%)$ \\
\hline Females, $\mathrm{n}(\%)$ & $413(52.81 \%)$ \\
\hline Age, Mean \pm SD (Range) & $37.3 \pm 8.99,(18-65)$ \\
\hline$\geq 40$ Years, $\mathrm{n}(\%)$ & $277(35.42 \%)$ \\
\hline$<40$ years, $\mathrm{n}(\%)$ & $505(64.57 \%)$ \\
\hline Duration of symptoms, & \\
$\geq 1$ year, $\mathrm{n}(\%)$ & $127(16.24 \%)$ \\
$<1$ year, $\mathrm{n}(\%)$ & $655(83.75 \%)$ \\
\hline
\end{tabular}

Table II: Stratification of QOL with respect to age, gender, BMI, smoking, DM, HTN and duration of symptoms $n=782$.

\begin{tabular}{l|c|c|c}
\hline $\begin{array}{l}\text { Characteristics with } \\
\text { total number }\end{array}$ & QOL impaired & QOL not impaired & p-value \\
\hline $\begin{array}{l}\text { Age (years) } \\
<40(505)\end{array}$ & $130(25.74 \%)$ & $375(74.25 \%)$ & 0.001 \\
$>40(277)$ & $169(61.02 \%)$ & $108(39.98 \%)$ & \\
\hline $\begin{array}{l}\text { Gender } \\
\text { Male (369) }\end{array}$ & $153(41.46 \%)$ & $216(58.5 \%)$ & 0.058 \\
$\quad$ Female (413) & $144(34.86 \%)$ & $269(65.13 \%)$ & \\
\hline BMI (Kg/m²) & $185(33.39 \%)$ & $369(66.60 \%)$ & 0.001 \\
$<25(554)$ & $112(49.12 \%)$ & $116(50.87 \%)$ & \\
$>25(228)$ & $103(78.04 \%)$ & $29(21.96 \%)$ & 0.001 \\
\hline Smoking & $194(29.8 \%)$ & $456(70.15 \%)$ & \\
Yes (132) & $61(74.39 \%)$ & $21(25.6 \%)$ & 0.001 \\
No (650) & $236(33.72 \%)$ & $464(66.28 \%)$ & \\
\hline DM & & & \\
Yes (82) & $54(85.7 \%)$ & $9(14.28 \%)$ & 0.001 \\
No (700) & $243(33.79 \%)$ & $476(66.2 \%)$ & \\
\hline HTN & $209(31.90)$ & $446(68.09 \%)$ & 0.001 \\
Yes (63) & $88(69.2 \%)$ & $39(30.70 \%)$ & \\
No (719) & & & \\
\hline Duration of symptoms & & & \\
$<1$ year (n=655) & & & \\
$\geq 1$ year (n=127) & & & \\
\hline & & & \\
\hline
\end{tabular}


correlated with age $>40$ years $(r=0.34, p=0.001)$, body mass index $(\mathrm{BMI})>25 \mathrm{Kg} / \mathrm{m}^{2}(\mathrm{r}=0.14, \mathrm{p}=0.001)$, history of smoking ( $r=-0.37, p=0.0001)$, and those suffering from DM $(r=-0.25, p=0.001)$ and hypertension $(r=-0.29$, $p=0.001$ ), respectively.

Stratification with respect to duration of disease also showed significant correlation $(r=0.024, p=0.001)$. However, no statistically significant correlation was observed with respect to ethnicity $(r=0.001, p=0.98)$. On logistic regression analysis, age more than 40 years, history of smoking, hypertension and DM showed statistically significant correlation with $p=<0.001$.

\section{DISCUSSION}

GERD is one of the commonest chronic gastrointestinal disorders, with esophageal and extra-esophageal symptoms and it can affect the QOL of patients.8,11-14 Despite diversity in the prevalence of the disorder, a major impact of GERD on QOL of patients has been shown worldwide. ${ }^{11,14,15}$

Effect of GERD on QOL has been evaluated in different studies by utilising questionnaires. Thirty-six-item ShortForm (SF-36) General Health Survey (SF-36) is a selfreported, generic health-related QOL (HRQOL) survey widely used in various chronic diseases including GERD. ${ }^{9}$ There are also other validated disease-specific tools to asses quality of life in GERD patients including gastrointestinal symptom rating scale (GSRS); reflux disease questionnaire (RDQ); quality of life in reflux and dyspepsia (QOLRAD) and GIS. 17

Alam et al. determined the impact of GERD on QOL at a single large public sector hospital in Karachi, Pakistan. The authors found poor QOL in $47 \%$ of patients. However, the results of only one study cannot be generalised to whole population and there was a need for more studies to assess the magnitude of impairment of QOL due to GERD. ${ }^{18}$ In the present study, QOL was impaired in $38 \%$ of patients having GERD. The difference may be secondary to inclusion criteria, as the earlier study included patients aged up to 40 years and duration of disease was specified to be 6 weeks to 6 months. Although in this study, only 277 (35.42\%) patients were more than 40 years; but it showed statistical significant association with impaired QOL.

Butt et al. studied the risk factors and prescription pattern in patients with GERD and showed that $46 \%$ of patients had $\mathrm{BMI} \geq 25 \mathrm{Kg} / \mathrm{m}^{2}, 19$ while in this study, $29.15 \%$ patients having GERD had $\mathrm{BMI} \geq 25 \mathrm{Kg} / \mathrm{m}^{2}$. Earlier study has not compared QOL with respect to BMI; however, in this study, no statistically significant association was documented with impaired QOL in multivariate regression analysis with respect to increased BMI.

A study published recently revealed that diabetes affects QOL and its impact increases when complications from diabetes occur. 20 In this study, about $10 \%$ of patients were diabetic; most of them had impaired QOL showing significant $(p=0.001)$. However, the present research did not assess the current status of diabetes control in the present study. Piper et al. noted that those, who quit smoking, reported a greater feeling of subjective wellbeing, which could eventually be used to lead them in quitting smoking. ${ }^{21}$ Striking similarity was observed in this study that those who were smokers had poor QOL. Carvalho et al. studied the effect of hypertension on HR-QOL and showed poorer QOL in hypertensive patients. ${ }^{22}$ Similar were the results in this study. Though in this study, only 20 patients were hypertensive but statistically significant $(p=0.001)$ association was found with impaired QOL.

Duration of disease also had significant impact on QOL in this study; 39 patients (14.02\%) had disease duration of $>1$ year and $74.35 \%$ (29 patients) of them had impaired QOL.

To the authors' knowledge, this is the largest study on Pakistani population with GERD and the impact of GERD on QOL of affected patients. The authors also report the impact of DM, hypertension and smoking on QOL in patients with GERD, modifying these factors may play a significant role in patient management. It was not objectively assessed the control of diabetes and hypertension as well as the number of cigarettes smoked per day; so in the future studies, there is a need to use the uniform protocol to assess the factors affecting QOL.

\section{CONCLUSION}

A significant proportion of patients with GERD have impaired QOL. Factors affecting QOL in such patients were higher age, history of smoking, hypertension and DM.

\section{ETHICAL APPROVAL:}

Ethical approval was sought from the Institutional Ethics Committee.

\section{PATIENTS' CONSENT:}

Written informed consent was obtained from all patients.

\section{CONFLICT OF INTEREST:}

Authors declared no conflict of interest.

\section{AUTHORS' CONTRIBUTION:}

GL, FMH: Concept of study, designed the work and collected data,

$\mathrm{AL}, \mathrm{MMH}$ : Performed acquisition of data processing, analysis and interpretation.

AAT, NHL: Reviewed and critically revised the draft.

\section{REFERENCES}

1. Philip OK, Lauren BG, Marcelo F. Guidelines for the diagnosis and management of gastroesophageal reflux disease. $\mathrm{Am} \mathrm{J}$ Gastroenterol 2013; 108:308-28. 
2. Vakil N, van Zanten SV, Kahrilas P, Dent J, Jones R. The Montreal definition and classification of gastroesophageal reflux disease: A global evidence-based consensus. Am J Gastroenterol 2006; 101:1900-20.

3. Feldman M, Friedman L, Brandt L. Sleisenger and Fordtran's Gastrointestinal and Liver Disease 9th Ed. New York: Elsevier; 2010: p.705-26

4. Spechler SJ, Jain SK, Tendler DA, Parker RA. Racial differences in the frequency of symptoms and complications of gastroesophageal reflux disease. Aliment Pharmacol Ther 2002; 16:1795-800.

5. Karim S, Jafri W, Faryal A, Majid S, Salih M, Jafri F, et al. Regular post-dinner walk: Can be a useful lifestyle modification for gastroesophageal reflux. J Pak Med Assoc 2011; 61:526-30.

6. Jafri N, Jafri W, Yakoob J, Islam M, Manzoor S, Jalil A, et al. Perception of gastroesophageal reflux disease in urban population in Pakistan. J Coll Physicians Surg Pak 2005; 15:532-4.

7. Dubois RW, Aguilar D, Fass R, Orr WC, Elfant AB, Dean BB, et al. Consequences of frequent nocturnal gastrooesophageal reflux disease among employed adults: Symptom severity, quality of life and work productivity. Aliment Pharmacol Ther 2007; 25:487-500.

8. McHorney CA, Ware Jr JE, Lu JF, Sherbourne CD. The MOS 36 -item short-form health survey (SF-36). III. Tests of data quality, scaling assumptions, and reliability across diverse patient groups. Med Care 1994; 32:40-66.

9. Jones $\mathrm{R}$, Coyne $\mathrm{K}$, Wiklund I. The gastrooesophageal reflux disease impact scale: A patient management tool for primary care. Aliment Pharmacol Ther 2007; 25:1451-9.

10. Sinha R, van den Heuvel WJ, Arokiasamy P. Validity and reliability of MOS short form health survey (SF-36) for use in India. Indian J Community Med 2013; 38:22-6.

11. Tack J, Becher A, Mulligan C, Johnson DA. Systematic review: The burden of disruptive gastrooesophageal reflux disease on health-related quality of life. Aliment Pharmacol Ther 2012; 35: 1257-66.

12. Eloubeidi MA, Provenzale D. Health-related quality of life and severity of symptoms in patients with Barrett's esophagus and gastroesophageal reflux disease patients without Barrett's esophagus. Am J Gastroenterol 2000; 95:1881-7.

13. iklund I, Carlsson J, Vakil N. Gastroesophageal reflux symptoms and well-being in a random sample of the general population of a Swedish community. Am J Gastroenterol 2006; 101:18-28.

14. Becher A, El-Serag H. Systematic review: The association between symptomatic response to proton pump inhibitors and health-related quality of life in patients with gastrooesophageal reflux disease. Aliment Pharmacol Ther 2011; 34: 618-27.

15. Fujiwara Y, Arakawa T. Epidemiology and clinical characteristics of GERD in the Japanese population. $J$ Gastroenterol 2009; 44:518-34.

16. Maleki I, Masoudzadeh A, Khalilian A, Daheshpour E. Quality of life in patients with gastroesophageal reflux disease in an Iranian population. Gastroenterol Hepatol Bed Bench 2013; 6:96-100.

17. Chan Y, Ching JY, Cheung CM, Tsoi KK, Polder-Verkiel S, Pang $\mathrm{SH}$, et al. Development and validation of a diseasespecific quality of life questionnaire for gastrooesophageal reflux disease: The GERD-QOL questionnaire. Aliment Pharmacol Ther 2010; 31:452-60.

18. Alam T, Moin F, Ali Z, Madiq M. Impact on quality of life in GERD patients using GERD impact scale. Gomal J Med Sci 2014; 12:97-100.

19. Butt AK, Hashemy I. Risk factors and prescription patterns of gastroesophageal reflux disease: HEAL study in Pakistan. J Pak Med Assoc 2014; 64:751-.57.

20. Trikkalinou A, Papazafiropoulou AK, Melidonis A. Type 2 diabetes and quality of life. World J Diabetes 2017; 8:120-9.

21. Piper ME, Kenford S, Fiore MC, Baker TB. Smoking cessation and quality of life: Changes in life satisfaction over 3 years following a quit attempt. Ann Behav Med 2012; 43:262-70

22. Carvalho MV, Siqueira LB, Sousa AL, Jardim PC. The influence of hypertension on quality of life. Arq Bras Cardiol 2013; 100:164-74. 